\title{
Ibuprofen, a Potential Cause of Acute Hemorrhagic Gastritis in Children - A Case Report
}

\author{
Maria Oana Mărginean ${ }^{1,2}$, Lorena Elena Meliț̦1,2*, Simona Mocanu³, Vlăduț Săsăran² \\ 1 Paediatrics Clinic 1, Târgu Mureș, Romania \\ 2 University of Medicine, Pharmacy, Sciences and Technology of Târgu Mureş, Romania \\ 3 Pathology Department County Clinic Hospital, Târgu Mureș, Romania
}

\begin{abstract}
Introduction: Upper gastrointestinal bleeding is an uncommon but possible life-threatening entity in children, frequently caused by erosive gastritis. Non-steroidal anti-inflammatory drugs are one of the most common class of drugs which can cause gastrointestinal complications, including hemorrhagic gastritis.

Case report: The case of a 6-year-old male, admitted for hematemesis, abdominal pain and loss of appetite. It was ascertained at the time of admission, that ibuprofen had been prescribed as the patient had a fever. This was inappropriately administered as the mother did not respect the intervals between the doses.

Initial laboratory tests revealed neutrophilia, leukopenia, high levels of lactate dehydrogenase and urea. An upper digestive endoscopy revealed an increased friability of the mucosa, digested blood in the gastric corpus and fornix. No active bleeding site was detected. The histopathological examination described a reactive modification of the corporeal gastric mucosa. Intravenous treatment with proton pump inhibitors and fluid replacement were initiated, with favorable results.
\end{abstract}

Conclusion: Ibuprofen can lead to upper digestive hemorrhage independently of the administered dose. Parents should avoid administering Ibuprofen for fever suppression without consulting their pediatrician.

Keywords: upper gastrointestinal bleeding, hemorrhagic gastritis, ibuprofen

Received: 16 April 2018 / Accepted: 03 October 2018

\section{INTRODUCTION}

Upper gastrointestinal bleeding can be a severe, lifethreatening condition in children, depending on the source, degree and cause of the hemorrhage. The most frequent sources of hematemesis are varices and mucosal gastric and duodenal lesions, often caused by drug ingestion [1]. Moreover, a Romanian study performed on 103 children discovered that the most frequent cause of upper gastrointestinal bleeding was erosive gastritis, which accounted for a third of all cases [2]. According to Deerojanawong et al. (2019) gastrointestinal bleeding is not uncommon among intensive care patients [3], with mechanically ventilated children being at risk of developing gastrointestinal hemorrhage [4].

In children, inadequate nutrition and drugs are cited as more common etiological factors [2,5], compared to adults, where gastrointestinal tumors represent a major cause of digestive bleedings.

Non-steroidal anti-inflammatory drugs (NSAIDs) are commonly used for their therapeutic effects which include analgesia, inflammation inhibition and fever suppression [6]. Although NSAIDs can lead to gastrointestinal complications, they are frequently prescribed worldwide [7]. Their primary mechanism of action consists of cyclooxygenase (COX) enzymes inhibition, thus reducing the synthesis of prostaglandins, which play a crucial role in gastroprotective mucus secretion [8]. Therefore, NSAIDs use can lead to several adverse gastrointestinal effects, such as gastric or duodenal ulcer, hemorrhage or perforation $[9,10]$. These complications are more common in non-selective NSAIDs, which act both on COX1 and COX2, whereas COX2selective inhibitors tend to lower the risk of gastrointestinal complications [8]. Although NSAIDs repre- 
sent the most common class of drugs which can cause gastrointestinal tract complications, other substances can also damage the gastric mucosa in children, such as corticosteroids, valproic acid or chemotherapeutic drugs. A rare case of hemorrhagic gastritis induced by oral iron supplements in a teenager female has also been described in the literature [11].

Ibuprofen, a non-selective COX inhibitor, is commonly used in pediatric patients, not only for its analgesic and antipyretic properties but also for safety reasons, in comparison with other drugs from its class. The guidelines concerning its posology and intervals of administration should be strictly respected in order to avoid adverse gastrointestinal effects [6].

This case report aims to underline the potential severe side-effects related to inappropriate administration of ibuprofen in children.

Informed written consent was obtained from the patient's mother before the publication of this case report.

\section{CASE REPORT}

\section{Presenting concerns and case history}

The case of a 6-year-old-male child, with no significant personal history, admitted to the emergency department of Pediatric Clinic 1, Târgu Mureş, Romania for vomiting, abdominal pain and loss of appetite. At the time of initial admission to the emergency department, it was reported by the parents that the child had experienced ten episodes of vomiting, four of which contained a significant quantity of fresh blood and three of them had the appearance of 'coffee ground'. According to the mother, the patient received three doses of $100 \mathrm{mg}$ ibuprofen, two hours apart from each other, followed by one dose of $190 \mathrm{mg}$ ibuprofen, six hours later. The latter dose was administrated arbitrarily by the mother, in the form of an oral suspension.

\section{Clinical findings}

The initial clinical examination indicated an altered general health status, pallor of the skin, productive cough, nasal obstruction, hyperemic pharynx and hypertrophic tonsils, mild abdominal tenderness in the epigastric area. The patient weighed $23 \mathrm{~kg}$.

\section{Diagnostic focus and assessment}

The initial laboratory tests revealed the following pathological elements: neutrophilia $(91.76 \%, 10850$ cells/ $\mu \mathrm{l})$, lymphocytopenia $(3.04 \%, 360$ cells/ $\mu \mathrm{l})$, high levels of lactate dehydrogenase $(269 \mathrm{U} / \mathrm{l})$. The hemoglobin $(12.7 \mathrm{~g} / \mathrm{dl})$ and hematocrit $(36.7 \%)$ levels were within normal ranges. After a twenty-four-hour period, the hemoglobin levels decreased following parenteral rehydration therapy, reaching a value of $10.3 \mathrm{~g} / \mathrm{dl}$.

An upper digestive endoscopy was performed within the following twelve hours after the admission, which revealed an increased friability of the mucosa, a medium quantity of digested blood in the gastric corpus and fornix; no active bleeding site was identified. These data corresponded to a Forrest II B classification of upper gastrointestinal hemorrhage, (Fig.1 and 2) indicating that no endoscopic intervention was needed. The histopathological examination from the gastric biopsy showed an antral gastric mucosa with lymphoid aggregates and reactive modifications of the corporeal gastric mucosa. There was no evidence of Helicobacter pylori (H. pylori) infection after microscopic evaluation of the gastric biopsies.

Based on these data, a final diagnosis was made of ibuprofen-induced hemorrhagic gastritis.

\section{Therapeutic focus and assessment}

Proton pump inhibitor therapy with Pantoprazol 20 mg (Takeda GmbH, Singen, Germany) was started intravenously immediately after admission in one daily dose and was maintained for five days. In order to compensate for the loss of fluids, perfusion with glucose and electrolytes was administered. The patient had two episodes of vomiting during the first night of hospitalization in which no signs of fresh or digested blood

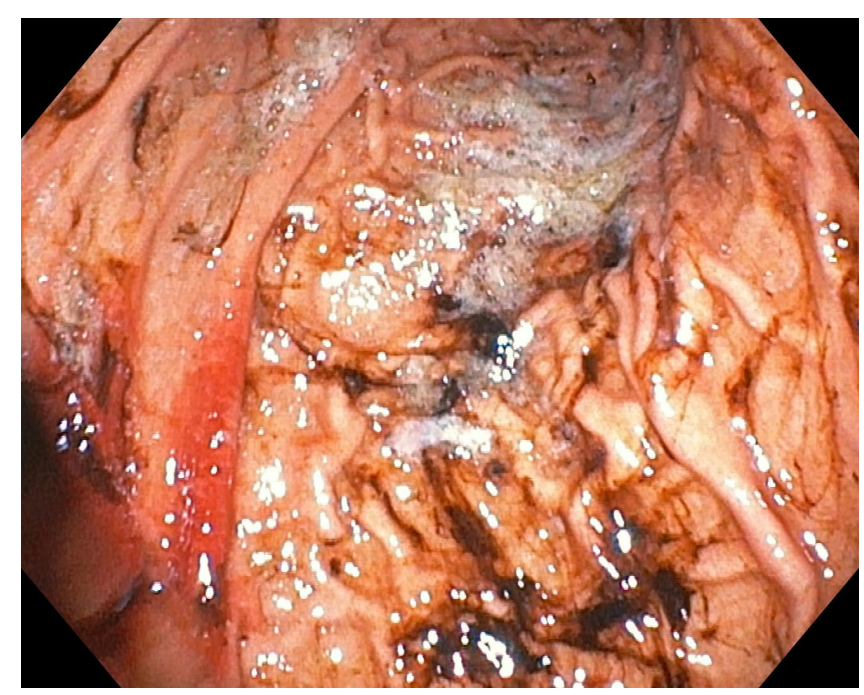

Fig. 1. The endoscopic aspect of digested blood in gastric corpus 
were detected. His evolution was favorable the next day, but his cough and rhinorrhea worsened towards the evening. Intravenous clindamycin (S.C. STADA M\&D, Timişoara, Romania), $230 \mathrm{mg}$, three times a day, together with nebulization with acetylcysteine (ZAMBON S.p.A., Vicenza, Italy) and concentrated saline solution $(\mathrm{NaCl} 3 \%)$, lead to an improvement in respiratory symptoms. The patient's condition slowly improved following this symptomatic treatment, though there was continuing persistent discomfort in the epigastric region accompanied by nausea. There was no recurrence of vomiting during the following two days.

The patient was discharged with the recommendation to continue with the clarithromycin $175 \mathrm{mg}$ (BGP Products S.R.L., Roma, Italy), two times a day for four days together with probiotics - Lactobacillus reuteri (Ewopharma International, Gothenburg, Sweden).

Oral proton-pump inhibitor therapy with $20 \mathrm{mg}$ of esomeprazole (AstraZeneca, GmbH, Wedel, Germany), once a day, was prescribed for thirty days. The adherence to a gastritis diet low in: fats, processed foods, fried meals and smoked products was advised.

\section{Follow-up and outcome}

The patient presented for a check-up one week after discharge. He had an excellent general status without any gastrointestinal complaints in the past week. Laboratory tests were carried out and showed no abnormal changes, except for a mildly elevated erythrocyte sedimentation rate (ESR), of $17 \mathrm{~mm} / \mathrm{h}$. Continuation of the prescribed treatment and diet was recommended.

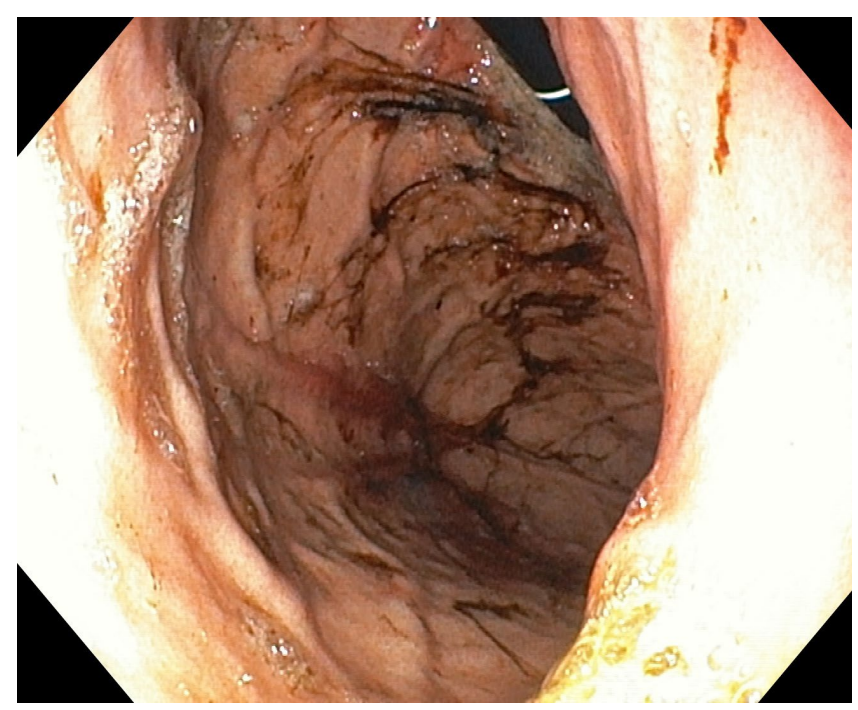

Fig. 2. The endoscopic aspect of digested blood in gastric corpus and fornix, but without an active bleeding site

\section{Discussions}

NSAIDs are generally considered safe drugs for fever management in pediatric cases if they are administered properly $[12,13]$. Ibuprofen is one of the most frequently used NSAIDs in pediatric cases due to its anti-inflammatory and antipyretic properties. The recommended posology for fever suppression is $5-10 \mathrm{mg} /$ $\mathrm{kg} /$ dose by mouth, every 6-8 hours, with a maximum total dose of $40 \mathrm{mg} / \mathrm{kg} /$ day [6]. In the above-described case, the patient had initially received three doses of ibuprofen, in lower amounts than the minimum recommended dose for fever control. However, the mother had not respected the intervals between the three initial doses, by administering oral ibuprofen every two hours. This could have been a risk factor for the adverse event that followed, although data in the literature describes several cases of gastrointestinal bleeding following intake of small doses of ibuprofen at appropriate intervals. Berezin et al. (2007) described a case series of four children, in which three of them received one dose of ibuprofen and one received two doses, and although the posology was appropriate, all of them developed hematemesis due to gastric antral ulcers [12]. Vaquero Sosa et al. (2013) reported on a study involving nine patients aged between 21 months and five years who had presented with upper gastrointestinal bleeding after two to four doses of ibuprofen. Only one of them had developed a superficial gastric ulcer, the others being diagnosed with acute hemorrhagic gastritis. Anemia or coagulopathies were not found in any of the children enrolled in the study, as was the case of our patient at the time of admission [14]. However, severe cases of gastrointestinal bleeding in children have also been published in the literature, Anyanwu et al. (2013) reported two cases of children presenting with melena associated with severe anemia. Both cases had a history of ibuprofen consumption in the past week, but with no precise quantification of the ingested doses. In one of the cases, a perforated ulcer was discovered, which required surgical intervention [15].

Additional risk factors can be associated with gastrointestinal bleeding in pediatric patients who had previously ingested NSAIDs, including a family history of peptic ulcers or H. pylori infection [9]. Genetic factors play in important role in children's' susceptibility to developing an $H$. Pylori infection, with various gene polymorphisms of interleukin 6 , tumor necrosis factor-alpha and angiotensin-converting enzyme, all increasing the risk of acquiring this bacterium [16]. 
A single-center study involving 1332 Romanian children reported a unique association between $\mathrm{H}$. pylori infection and acute hemorrhagic gastritis in eight cases [17]. Other recent reports in the literature describe the importance of $H$. pylori eradication in the evolution of patients with NSAIDs-induced acute hemorrhagic gastritis. [18] Histopathological examination did not identify the presence of $H$. pylori in the present case.

The risk stratification scores for upper gastrointestinal bleedings in children are still under development, and therefore the management of this pathology remains a challenge for pediatricians [19].

\section{CONCLUSIONS}

Upper gastrointestinal bleeding is a possible adverse outcome of flawed NSAIDs administration. Short interval administration of NSAIDs should be avoided due to possible dose accumulation. Parents should steer clear of administering NSAIDs without consulting their pediatrician regarding posology and intervals of NSAIDs intake.

\section{CONFLICT OF INTEREST}

None to declare.

\section{- REFERENCES}

1. Bhatia V, Lodha R. Upper gastrointestinal bleeding. Indian J Pediatr. 2011;78:227-33.

2. Gimiga N, Olaru C, Diaconescu S, Miron I, Burlea M. Upper gastrointestinal bleeding in children from a hospital center of Northeast Romania. Minerva Pediatr. 2016;68:189-95.

3. Reintam A, Parm P, Kitus R, Kern H, Starkopf J. Gastrointestinal symptoms in intensive care patients. Acta Anaesthesiol Scand. 2009;53:318-24.

4. Deerojanawong J, Peongsujarit D, Vivatvakin B, Prapphal N. Incidence and risk factors of upper gastrointestinal bleeding in mechanically ventilated children. Pediatr Crit Care Med. 2009;10:91-5.

5. Mureşan M, Bancu S, Bara T, Bancu L, Turcu M, Mureşan S. [Local recurrence after the sphincter-saving operations and abdominal perineal resection in rectal cancer]. Chirurgia (Bucur). 2009;104:415-8.

6. Dills R, Anderson LA, Pierce CA. The role of nonsteroidal anti- inflammatory drugs in pediatric patients. Pharmacol Res. 2012;65:5-8.

7. Brune K, Patrignani P. New insights into the use of currently available non-steroidal anti-inflammatory drugs. J Pain Res. 2015;8:105-18.

8. Rafaniello C, Ferrajolo C, Sullo MG et al. Risk of gastrointestinal complications associated to NSAIDs, low-dose aspirin and their combinations: Results of a pharmacovigilance reporting system. Pharmacol Res. 2016;104:108-14.

9. Cardile S, Martinelli M, Barabino A et al. Italian survey on nonsteroidal anti-inflammatory drugs and gastrointestinal bleeding in children. World J Gastroenterol. 2016;22:1877-83.

10. Lanza FL, Chan FKL, Quigley EMM, Practice Parameters Committee of the American College of Gastroenterology. Guidelines for prevention of NSAID-related ulcer complications. Am J Gastroenterol. 2009;104:728-38.

11. Meliţ LE, Mărginean CO, Mocanu S, Mărginean MO. A rare case of iron-pill induced gastritis in a female teenager: A case report and a review of the literature. Medicine (Baltimore). 2017;96:e7550.

12. Berezin SH, Bostwick HE, Halata MS, Feerick J, Newman LJ, Medow MS. Gastrointestinal bleeding in children following ingestion of low-dose ibuprofen. J Pediatr Gastroenterol Nutr. 2007;44:506-8.

13. Mărginean CO, Meliţ LE, Chinceşan M et al. Communication skills in pediatrics - the relationship between pediatrician and child. Medicine (Baltimore). 2017;96:e8399.

14. Vaquero Sosa E, Bodas Pinedo A, Maluenda Carrillo C. [Gastrointestinal bleeding following ingestion of low-dose ibuprofen]. An Pediatr (Barc). 2013;78:51-3.

15. Anyanwu LJC, Mohammad AM. Gastrointestinal bleeding following NSAID ingestion in children. Annals of Pediatric Surgery. 2013;9:87-9.

16. Mărginean MO, Mărginean CO, Meliţ LE, Voidăzan S, Moldovan V, Bănescu C. The impact of host's genetic susceptibility on Helicobacter pylori infection in children. Medicine (Baltimore). 2017;96:e7612.

17. Mărginean CO, Cotoi OS, Pitea AM, Mocanu S, Mărginean C. Assessment of the relationship between Helicobacter pylori infection, endoscopic appearance and histological changes of the gastric mucosa in children with gastritis (a single center experience). Rom J Morphol Embryol. 2013;54:709-15.

18. Lee S-Y. Future candidates for indications of Helicobacter pylori eradication: do the indications need to be revised? J Gastroenterol Hepatol. 2012;27:200-11.

19. Nasher O, Devadason D, Stewart RJ. Upper Gastrointestinal Bleeding in Children: A Tertiary United Kingdom Children's Hospital Experience. Children (Basel). 2017;4:95. 